\title{
Ocular manifestations of leishmaniasis in a cat: first case report from Brazil
}

\author{
[Manifestações oculares da leishmaniose em um gato: primeiro relato de caso do Brasil]
}

G. Madruga, A.P. Ribeiro*, T. Ruiz, V.R.F. Sousa, C.G. Campos, A.B.P.F. Almeida, C.A. Pescador, V. Dutra

Faculdade de Medicina Veterinária - Universidade Federal de Mato Grosso - Cuiabá, MT.

\begin{abstract}
An 8-year-old domestic short hair female cat initially presented with bilateral uveitis with pseudotumoral appearance. The patient tested negative for feline immunodeficiency virus (FIV), feline leukemia virus (FeLV) and Toxoplasma gondii. Histopathology of a granulomatous lesion on the upper left conjunctiva revealed amastigotes compatible with Leishmania spp. Aqueous humor was aspired and the diagnosis was confirmed after isolation of promastigotes cultivated in biphasic NNN medium and by positive polymerase chain reaction (PCR) for Leishmania infantum. Treatment with allopurinol (10mg/kg/ BID/PO) was commenced and a natural insect repellent was prescribed. Six months of treatment with allopurinol associated with the initial topical medications helped to improve ocular signs. Leishmaniasis should be considered as a differential diagnosis in cats presenting uveitis with pseudotumoral appearance. To our knowledge, this is the first report of feline leishmaniasis with ocular manifestation in Brazil, in which diagnosis was confirmed by aqueous humor analysis.
\end{abstract}

Keywords: cat, uveitis, Leishmania infantum, paracentesis

\section{RESUMO}

Uma gata, sem raça definida, de oito anos de idade, foi atendida inicialmente com uveíte bilateral, com aparência pseudotumoral em íris. Foi realizado teste para o vírus da imunodeficiência felina (FIV), da leucemia felina $(\mathrm{FeLV})$ e de Toxoplasma gondii, obtendo-se resultados negativos. $O$ exame histopatológico da conjuntiva superior do olho esquerdo revelou amastigotas compativeis com Leishmania spp. Foi realizada paracentese, e promastigotas foram isoladas no humor aquoso, cultivadas em meio NNN bifásica. Reação em cadeia da polimerase (PCR) confirmou diagnóstico positivo para Leishmania infantum. Tratamento com alopurinol $(10 \mathrm{mg} / \mathrm{kg} / \mathrm{BID} / \mathrm{PO})$ foi iniciado, e um repelente natural de insetos foi prescrito. Seis meses de tratamento com alopurinol associado aos medicamentos tópicos iniciais ajudaram a melhorar os sinais oculares. Leishmaniose deve ser considerada como um diagnóstico diferencial nos gatos que apresentam uveíte com aparência pseudotumoral de íris. Até o presente momento, este é o primeiro relato de leishmaniose felina com manifestação exclusivamente ocular da doença no Brasil cujo diagnóstico foi confirmado por meio de análise de humor aquoso.

Palavras-chave: gato, uveíte, Leishmania infantum, paracentese

\section{INTRODUCTION}

Leishmaniasis is a chronic zoonotic disease, which can potentially cause death (Pennisi et al., 2015). This disease is endemic along the Mediterranean coast, areas in East Africa, India, Central America, and South America (Pennisi et

Recebido em 28 de novembro de 2016

Aceito em 3 de fevereiro de 2017

*Autor para correspondência (corresponding author)

E-mail: alexandre.aleribs@gmail.com al., 2015). In the Americas, spreading occurs through sand flies of the genus Lutzomyia that transmit Leishmania infantum, a protozoan capable of causing the severe form of the disease (Almeida et al., 2012).

In Brazil, dogs were considered to be the only domestic reservoir of $L$. infantum until 2008 
(Silva et al., 2008). However, natural infection of domestic cats with Leishmania spp. has been reported in 4/27 Brazilian states (Pennisi et al., 2015).

To date, $L$. infantum is the most frequently isolated species in Brazil identified in cats and in several countries throughout the world, such as Spain, Italy, Iran, Switzerland, Portugal and Greece (Pennisi et al., 2015).

Most cats affected by visceral leishmaniasis exhibit mucocutaneous lesions and lymphadenomegaly (Pennisi et al., 2015). Ophthalmic abnormalities secondary to leishmaniasis infection have been described in approximately one third of affected cats (Pennisi et al., 2015). Unilateral or bilateral uveitis is the most common ophthalmic finding in these cases, and generally exhibits a granulomatous pseudotumoral pattern that may progress to panophthalmitis (Leiva et al., 2005, Ritcher et al., 2014).

This report aimed to describe general and ocular clinical signs of a cat with visceral leishmaniasis, in which the ophthalmic diagnose was confirmed by aqueous humor culture and PCR. In addition, to decribe the evolution of the clinical signs during a 6-months period. To our knowledge, this is the first report of feline leishmaniasis with ocular manifestation in Brazil.

\section{CASE REPORT}

An 8-year-old female domestic shorthair cat was presented at the Ophthalmology Service of the Veterinary Teaching Hospital of the Federal University of Mato Grosso, Cuiabá- MT, with a history of bilateral photophobia, blepharospasm, and conjunctival hyperemia for approximately 3 months. The owner denied previous treatment. Deworming and vacinations were updated. On physical examination, the patient exhibited good general condition, with no signs of malnutrition, skin disease, lymphadenomegaly, or abnormalities on abdominal palpation. All physiological parameters were within the normal ranges for the species.

On ophthalmic examination, the Schirmer tear test readings (Ophthalmos Ind. Farmacêutica, São Paulo, Brazil) were 8 and $10 \mathrm{~mm} / \mathrm{min}$ in the oculus dexter (OD) and oculus sinister (OS), respectively. Menace response, pupillary and dazzle reflexes were normal. Slit-lamp biomicroscopy (SL-14, Kowa Company, Tokyo, Japan) revealed moderate conjunctival hyperemia, chemosis, and mucoid discharge in both eyes. The upper eyelid of the OS exhibited conjunctival thickening without ulcerations. Stromal keratitis was observed on the dorsal temporal region of the same eye, with loss of limbal structure in this area. Additionally, thickening of the iris on the dorsal temporal region of the OS was observed (Figure 1-a). The cornea of the OD exhibited mild edema and neovascularization; moderate aqueous flare, and thickening of the iris with a pseudotumoral appearance, which was more intense on the dorsal temporal region were seen (Figure 2-a). Intraocular pressure (IOP) was assessed with applanation tonometry (Tonopen-XL, Mentor O $\& \mathrm{O}$, Inc.) and was 11 and $12 \mathrm{mmHg}$ for the OD and OS, respectively. After pupil dilation with $1 \%$ tropicamide (Mydriacyl®) no abnormalities were seen in the lens, vitreous, and the retina of both eyes. Fluorescein dye test was negative in both eyes. Ocular ultrasound (MyLab ${ }^{\mathrm{TM}}$ Five Vet; Esaote Pie Medical, Genova, Italy) performed with a $10 \mathrm{MHz}$ linear transducer revealed homogeneous hyperechoic thickening of the iris in the OD, and absence of alterations in the remaining intraocular and retrobulbar structures.

Results of complete blood cell count, serum levels of alanine aminotransferase (ALT), albumin, creatinine, and urea, were within the reference range for the species. Thoracic radiographs, abdominal ultrasound, and echocardiogram were unremarkable. AntiToxoplasma gondii specific IgG and FIV/FeLV rapid test (Alere $\mathrm{TM}^{\circledR}$, Bionote, Gyeonggi-do, Korea) were negative.

Based on the clinical history and ocular examination that revealed anterior uveitis and thickening of the iris, uveal neoplasm was suspected, and anterior chamber paracentesis was suggested to confirm the diagnosis. Following general anestesia with propofol, $0.2 \mathrm{~mL}$ of aqueous humor was collected from both of eyes. Aqueous humor cytopathology stained with modified Wrights stain revealed mixed inflammatory cell content but neoplastic cells were not detected. 
Ocular therapy was initiated with $0.3 \%$ ketorolac trometamol (Acular®) and $1 \%$ prednisolone acetate (Predfort ${ }^{\circledR}$ ) every $6 \mathrm{~h}$, for 10 days. In the first recheck the patient showed no signs of improvement of the ocular signs. A biopsy of the eyelid and palpebral conjunctiva of the OS, and a new anterior chamber paracentesis of both eyes was suggested for further investigation. In addition, blood sample was collected from the jugular vein, and bone marrow sample was collected from the esternun.

Since general anesthesia was required, the owner requested elective ovariohysterectomy. During the procedure, enlarged mesenteric lymph node was detected and a biopsy specimen was collected, placed in $10 \%$ neutral buffered formalin and submitted for histological analysis.

The sections of the eyelid and palpebral conjunctiva stained with $H \& E$ revealed the presence of inflammatory infiltrate on the superficial dermis composed mainly of histiocytes, with basophilic intracytoplasmic and extracytoplasmic amastigotes compatible with the protozoan Leishmania spp. (Figure 3-a). Examination of the mesenteric lymphnode sample stained with $\mathrm{H} \& \mathrm{E}$ revealed many macrophages containing multiple amastigotes that were also compatible with the protozoan Leishmania spp. (Figure 3-b).

In order to confirm the involvement of Leishmania spp. as a causative agent of anterior uveitis, samples of aqueous humor, bone marrow, and blood were seeded in biphasic NNN medium, and promastigotes were isolated from all samples. Additionally, PCR from samples of aqueous humor, blood, and bone marrow revealed the presence of Leishmania spp. DNA. Sequencing showed a 99\% homology to a sequence of $L$. infantum in GenBank (accession number XR_001203206.1, FR799614.1, GQ332356.1, M81430.1) (Figure 4).
Once the diagnosis of leishmaniasis was estabilished, treatment with allopurinol $(10 \mathrm{mg} / \mathrm{kg} / \mathrm{BID} / \mathrm{PO})$ was commenced and a natural insect repellent was prescribed. The previous ophthalmic treatment was continued, once improvement of the ocular signs were not observed until the second recheck, 10 days latter.

In the third recheck, 15 days from the beginning of the leishmaniasis specific treatment, the iris slowen of the OD and the chemosis of the OS showed slighty improvement. Mild aqueous flare was still observed in both of eyes and IOPs were 13 and $12 \mathrm{mmHg}$ for the OD and OS, respectively. Topical treatment was maintained for 3 more months, with ophthalmic evaluations performed every 15 days, once the owner reported that the patient exhibited blepharospasm and loss of appetite when the ocular medications were withdrawn. Following 30 days of treatment with allopurinol and topical treatment, the OD developed mild iris atrophy but remained calm (Figure 1-b), and the OS showed improvement of conjunctival thickening, loss of limbal delimitation, still exhibited intense corneal vascularization, and corneoscleral melanosis (Figure 2-b). At this time, IOP were 10 and $13 \mathrm{mmHg}$ for the OD and OS, respectively.

In the last recheck, which occured 6 months later from the first appointment, the prednisolone acetate eye drops (Predfort (B) was still being used every $12 \mathrm{~h}$. During this period, complete blood count revealed leukopenia $\left(5000 \mathrm{~mm}^{3}\right)$ and thrombocytopenia $\left(78.000 / \mathrm{mm}^{3}\right)$. Renal and hepatic function test results were within the normal range. Specific tests for leishmaniasis were performed: enzyme-linked immunoassay (ELISA) was non-reagent and indirect immunofluorescence (IFI) test was considered negative $(<1: 40)$. No signs of anterior uveitis were observed in both of eyes at this time and IOP was 11 and $12 \mathrm{mmHg}$ for the OD and OS, respectively. 

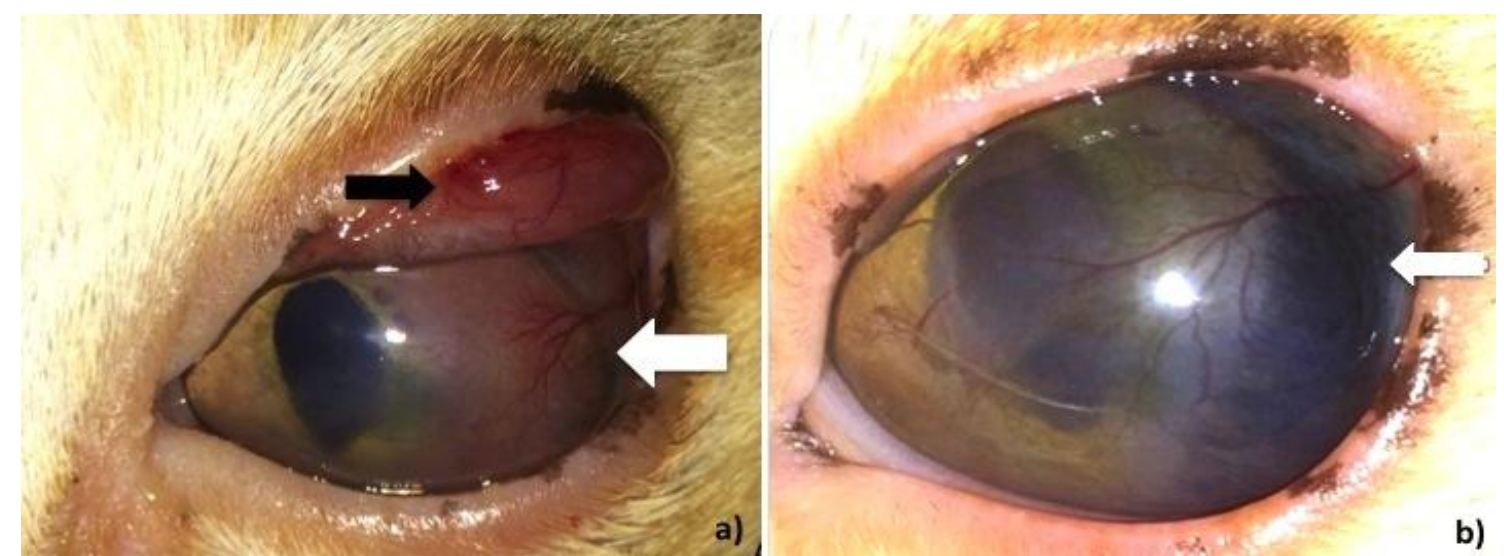

Figure 1. Ophthalmic findings of feline leishmaniasis. A- oculus sinister. Upper eyelid conjunctival thickening (black arrow). Note non-ulcerative keratitis with intense neovascularization (white arrow) associated with moderate uveitis. B- left eye after 3 months of ocular treatment. Note improvement of conjunctival thickening, loss of limbal delimitation, still exhibiting intense neovascularization, and corneoscleral melanosis (white arrow).
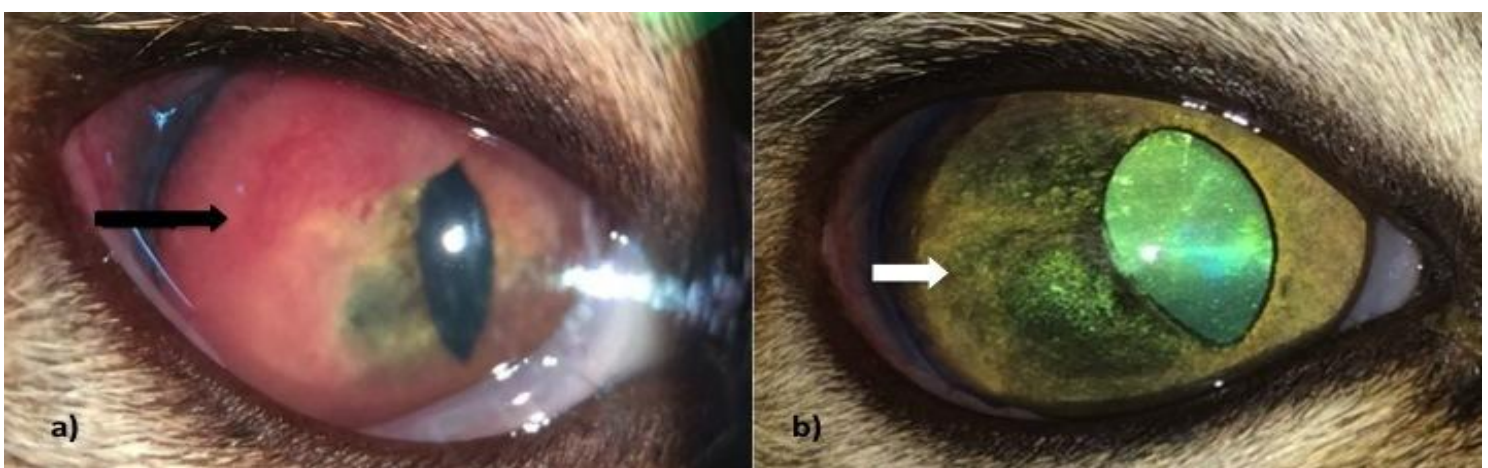

Figure 2. A - oculus dexter. Pseudotumoral appearance of the iris on the dorsal temporal region (black arrow). Note severe uveitis with conjunctival hyperemia, moderate flare, and miosis. B- right eye after 3 months of ocular treatment. Significant improvement of uveitis, remission of iridic neovascularization and development of mild iris atrophy (white arrow).

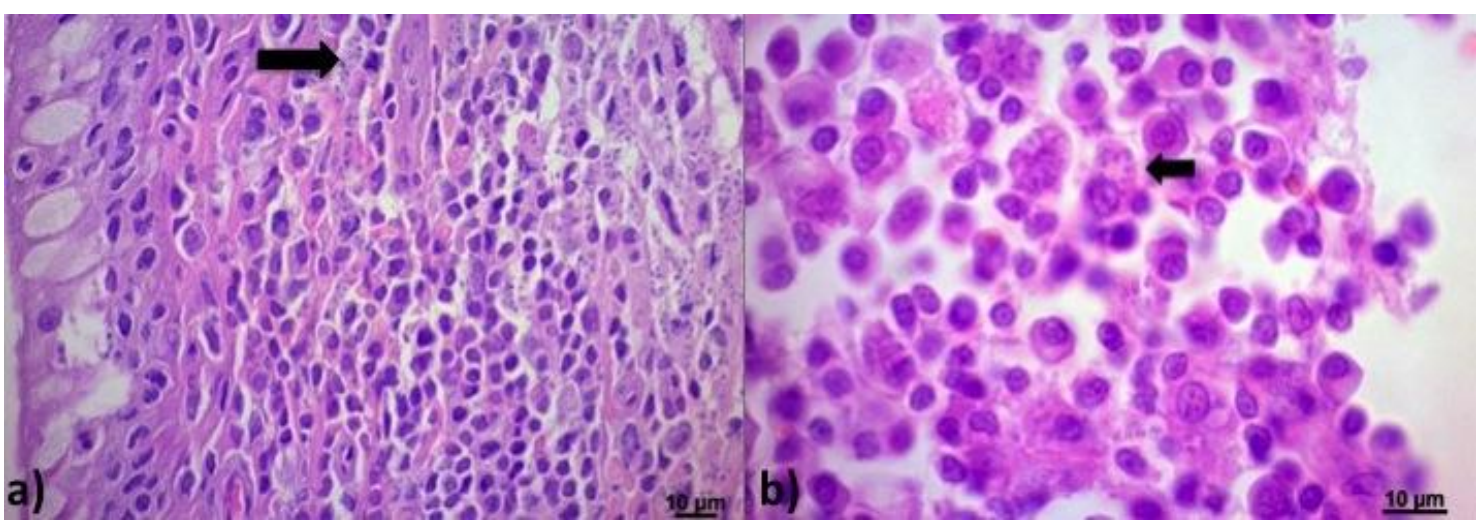

Figure 3. A- Histological section of palpebral conjunctiva of the oculus sinister. Presence of inflammatory infiltrate composed mainly of histiocytes in the superficial dermis of the eyelid, with basophilic intracytoplasmic and extracytoplasmic amastigotes compatible with the protozoan Leishmania spp. (arrow). B- Mesenteric lymph node containing a large number of macrophages with multiple intracytoplasmic amastigotes compatible with the protozoan Leishmania spp. (arrow). 


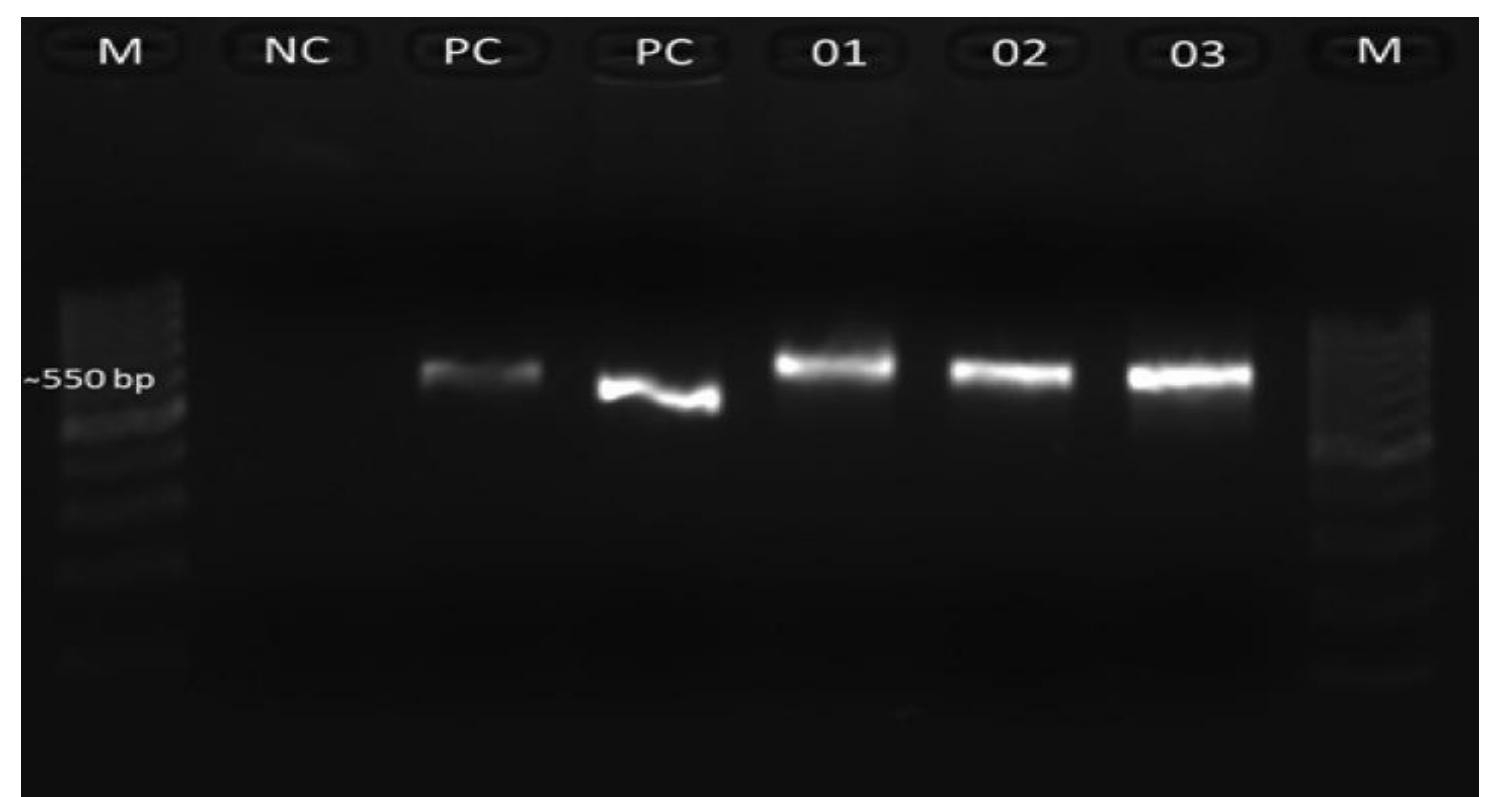

Figure 4. Agarose gel electrophoresis showing the amplified products with samples of the cat. M: DNA ladder 100pb; NC: negative control; PC: positive controle 600bp (DNA control of L. infantum (MHOM/BR/1974/PP75); PC: positive controle (DNA control of L. infantum (LL 1489); Lane 1: Aqueous humor sample of cat; Lane 2: blood sample of cat; Lane 3: skin sample of cat.

\section{DISCUSSION}

The most common clinical signs of leishmaniasis in cats described in the literature include lesions of the skin, mucocutaneous junctions, and lymphadenomegaly (Pennisi et al., 2015). The possibility of finding amastigotes of Leishmania spp. in cats through cytology or histology of liver, spleen, lymph nodes, stomach, large intestine, kidney, oral mucosa, nasal exudate, and ocular tissues has been reported (Leiva et al., 2005, Sobrinho et al., 2012, Pennisi et al., 2015). However, in most cats infected by leishmaniasis, clinical signs have been correlated with other causes including retroviral infection (FIV and FeLV), immunosuppressive treatment, and debilitating concomitant diseases such as malignant neoplasia, diabetes mellitus, toxoplasmosis, and haemobartonellosis (Hervás et al., 2001, Leiva et al., 2005, Sobrinho et al., 2012, Pennisi et al., 2015). In the patient of the present report, these diseases were excluded by physical examination and laboratory findings.

In the case described here, visible clinical manifestations were exclusively ophthalmic, and the mesenteric lymphadenomegaly was only noticed during ovariohysterectomy. To date, only two cases of feline leishmaniasis with exclusively ocular manifestations, namely uveitis and blepharitis, had been described in the literature (Verneuil, 2013, Ritcher et al., 2014,). In other reports, patients exhibited other signs in addition to ocular signs, such as stomatitis, anorexia, dermatitis, and alopecia in certain areas of the body (Hervás et al., 2001, Leiva et al., 2005).

In cats, amastigotes have been found by cytology in samples of conjunctiva (Navarro et al., 2010), cornea (Leiva et al., 2005), uveal tract (Hervás et al., 2001, Leiva et al., 2005), and aqueous humor (Pennisi et al., 2015). However, in the paper of Pennisi et al. (2015), aqueous humor cytolopathological findings were not reported. The cytopathologic result of our first aqueocentesis showed mixed inflammatory cell content, which it was not elucidative for a conclusive diagnosis. Although aqueous humor culture from the second paracentesis had revealed the presence of promastigotes. Therefore, we performed aqueous humor PCR to confirm the intraocular involvement of $L$. infantum.

Unlike other cases of uveitis caused by feline leishmaniasi, our patient did not develop increased IOP and secondary glaucoma (Hervás 
et al., 2001, Leiva et al., 2005, Verniuil, 2013, Ritcher et al., 2014). Pennisi et al. (2015) reported that ocular manifestations such as uveitis, blepharitis, and conjunctivitis have been described in cases of feline visceral leishmaniasis that usually exhibit other systemic clinical signs. However, in the present report, only bilateral uveitis unresponsive to anti-inflammatory therapy was observed. The patient did not exhibit any other apparent clinical sign 3 months after the diagnosis of leishmaniasis. Blepharitis and keratoconjunctivitis have also been described as the most frequent lesions in cats with leishmaniasis (Navarro et al., 2010, Migliazzo et al., 2015); however, granulomatous anterior uveitis with pseudotumoral appearance was the predominant clinical sign in this report and in others (Hervás et al., 2001, Leiva et al., 2005, Verniuil, 2013, Ritcher et al., 2014).

Long treatments with allopurinol or subcutaneous injections of meglumine antimoniate have been routinely used for cats with leishmaniasis despite the lack of information on the pharmacokinetics of these drugs (Pennisi et al., 2015). Although such agents are well tolerated, frequent monitoring of renal and hepatic function is imperative during management of the disease (Pennisi et al., 2015). Following 3 months of treatment with allopurinol and topical anti-inflammatory therapy, renal and hepatic functions of the patient were within normal ranges. Nonetheless, complete blood count revealed leukopenia and thrombocytopenia, which are commonly found in dogs but not in cats with this disease (Pennisi et al., 2015). In the last recheck, the patient tested negative to ELISA and IFI were $<1: 40$, which is considered negative.

Since there are no studies regarding the use of vaccines for leishmaniasis in cats, the best prevention strategy for this species is the use of topical inseticides with repellent activity similar to those applied on dogs. Unfortunately, permethrin and deltamethrin should not be used in cats owing to their toxicity (Pennisi et al., 2015). In the case reported here, the patient was using a low toxicity natural citronella phlebotomine repellent formulated for animals, which is used as a topical repellent in humans; however, its efficacy still warrants further investigation in cats (Warburg and Faiman, 2011).
Allopurinol therapy has been reported to significantly aid in the remission of clinical signs of uveitis in a cat with leishmaniasis (Ritcher et al., 2014). In the report of Ritcher et al. (2014), the patient exhibited ocular improvement 2 months following allopurinol therapy and topical medication (prednisolone combined with dorzolamide and timolol). In the present report, OS of our patient did not shown signs of improvement of uveitis, despite of maintenance of ophthalmic eye drops (ketorolac trometamol and prednisolone) combined with systemic allopurinol. In cats, there are no studies concerning the effects of allopurinol on intraocular inflammation caused by infection by Leishmania spp.

In conclusion, this report presents the first description of leishmaniasis with exclusively ocular clinical manifestation in a cat in Brazil. Furthermore, this is the first confirmed report of leishmaniasis in a cat by aqueous humor culture and PCR, which highlights the importance of these methods as supportive tools for the diagnosis of leishmaniasis - associated uveitis in cats.

\section{REFERENCES}

ALMEIDA, A.B.P.F.; PAULA, D.A.J. et al. Characterization of canine leishmaniasis by PCR-RFLP in Cuiaba, Mato Grosso, Brazil. Arch. Vet.Sci., v.17, p.68-72, 2012.

HERVÁS, J.; CHÁCON-MANRIQUE DE LARA, F.; LÓPEZ, J. et al. Granulomatous (pseudotumoral) iridociclitis associated with leishmaniasis in a cat. Vet. Record, v.149, p.624$625,2001$.

LEIVA, M.; LLORET, A.; PENA, T. et al. Therapy of ocular and visceral leishmaniasis in a cat. Vet. Ophthalmol., v.8, p.71-75, 2005.

MIGLIAZZO, A.; VITALE, F.; CALDERONE, S. et al. Feline leishmaniosis: a case with a high parasitic burden. Vet.Dermatol., v.26, p.69-70, 2015.

NAVARRO, J.A.; SÁNCHEZ, J.; PEÑAFIELVERDÚ, C. et al. Histopathological lesions in 15 cats with leishmaniosis. J. Comp.Pathol., v.143, p.297-302, 2010. 
PENNISI, M.G.; CARDOSO. L.; BANETH, G. et al. LeishVet update and recommendations on feline leishmaniosis. Parasit.Vectors, v.8, p.302, 2015.

RICHTER, M.; SCHAARSCHMIDT-KIENER, D.; KRUDEWIG, C. Ocular signs, diagnosis and long-term treatment with allopurinol in a cat with leishmaniasis. Schweiz Arch Tierheilkd, v.156, p.289-294, 2014.

SILVA, A.V.M; CANDIDO, C.D.S.; PEREIRA, D.P. et al. The first record of American visceral leishmaniasis in domestic cats from Rio de Janeiro, Brazil. Acta Trop., v.105, p.92-94, 2008.
SOBRINHO, L.S.; ROSSI, C.N.; VIDES, J.P. et al. Coinfection of L. infantum with Toxoplasma gondii, Feline Immunodeficiency Virus (FIV) and Feline Leukemia Virus (FeLV) in cats from an endemic area of zoonotic visceral leishmaniasis. Vet. Parasitol., v.187, p.302-306, 2012.

VERNEUIL, M. Leishmaniose oculaire féline: à propos d'uncas. J. Fr. Ophtalmol., v. 36, p.6772, 2013 .

WARBURG, A.; FAIMAN, R. Research priorities for the control of phlebotomine sand flies. J. Vector Ecol., v.36, p.10-16, 2011. 